appropriate in general for stable or follow-up patients in comparison to new or acutely unwell patients. There was some worry expressed about missing out non-verbal cues which assist with mental state examination.

Conclusion. Inspite of a low response rate, the survey provided some understanding about the experience of doctors practicing tele-psychiatry during pandemic. While technological challenges were acknowledged, tele-psychiatry seemed to have been accepted by a majority of doctors who are also willing to continue it in their future clinical practice. There is a need to explore in a larger sample involving both patients and clinicians about the beneficial effects of tele-psychiatry that can be incorporated in the usual psychiatric practice.

\section{Revisiting vitamin D status and supplementation for inpatients with intellectual and developmental disability in the north of England, UK}

Vlad Ciausu $^{1 *}$, Marcin Ostrowski ${ }^{1}$, Bethany Dudley ${ }^{1}$, Chris Ince ${ }^{1}$ and Iain McKinnon ${ }^{2}$

${ }^{1}$ Cumbria, Northumberland, Tyne And Wear NHS Foundation Trust and ${ }^{2}$ Newcastle University, Cumbria, Northumberland, Tyne And Wear NHS Foundation Trust

${ }^{*}$ Corresponding author.

doi: 10.1192/bjo.2021.95

Aims. Vitamin D deficiency is common among people with Intellectual and Developmental Disability (IDD) and is linked to worse health outcomes.

Our aims were to re-evaluate vitamin $\mathrm{D}$ testing and supplementation among inpatients with IDD, examine any correlates with physical health conditions including COVID-19 and make recommendations for the current regime of supplementation and testing within inpatient IDD services.

Method. The study population comprised inpatients who were in any of the Northgate Hospital IDD inpatient services in Northumberland, UK. The wards sampled were the Medium Secure Unit, Low Secure Unit, Hospital Based Rehabilitation Wards and Specialist Autism Inpatient Service. Records of all inpatients between January 2019 and July 2020 were examined for 25-hydroxyvitamin D [25(OH)D] level, ward area, supplementation status, test seasonality, medication, and health status.

We performed a correlation to see whether there was an association between vitamin $\mathrm{D}$ level and length of time on treatment. In addition, comparison of the replete and inadequate group for age, ethnicity, seasonality, ward location and psychotropic medication was undertaken.

Data on physical health risk factors, obesity and COVID-19 infection were also collected. The physical comorbidities were described in order to evaluate whether any emerging patterns relating to COVID-19 infection were emerging.

Result. There were 67 inpatients in Northgate IDD services on 1 January 2019, with 11 further patients admitted up to the end of the sampling period on 31 July 2020. Nineteen patients were discharged during that period, so the sample comprised 78 patients.

Ages were comparable across three of the ward areas, except for an older group of patients in the hospital-based rehabilitation setting. Mean 25(OH)D level for supplemented (800IU/day) patients was $75 \mathrm{nmol} / \mathrm{l}$ (SD 20) compared to $40 \mathrm{nmol} / \mathrm{l}$ (SD 19) in the nonsupplemented group $(\mathrm{p}<0.001)$.

Thirty-eight percent of those who were inpatients during the first wave of the COVID-19 pandemic developed symptoms, but the small sample size could not establish vitamin D levels as a predictor of outcome.
Conclusion. Our findings show that clinicians continue to offer vitamin D supplementation for inpatients, at a dose of $800 \mathrm{IU}$ $(20 \mu \mathrm{g})$ per day.

The mean vitamin D levels we observed were higher for those on supplements compared to our 2013 baseline data, whereas patients not on supplementation now had levels akin to those found previously. Vitamin D (800IU/day) supplementation is effective but adequacy of the nationally recommended dose of 400IU/day is unclear. Links to COVID-19 merit further research.

\section{Investigating Transcranial Direct Current Stimulation} (TDCS) in obsessive compulsive disorder (OCD): a double-blind, sham-controlled, cross-over randomised trial

Eduardo Cinosi ${ }^{1 \star}$, David Baldwin ${ }^{2}$, Tim Gale ${ }^{1}$, Matt Garner ${ }^{2}$, Natalie Hall ${ }^{3}$, Daniel Meron ${ }^{4}$, Nick Sireau ${ }^{5}$, David Wellsted ${ }^{3}$, Solange Wyatt ${ }^{3}$ and Naomi A Fineberg ${ }^{6}$

${ }^{1}$ Hertfordshire Partnership University NHS Foundation Trust, University of Hertfordshire; ${ }^{2}$ University of Southampton; ${ }^{3}$ University of Hertfordshire; ${ }^{4}$ University of Southampton, Somerset Partnership NHS Foundation Trust; ${ }^{5}$ ORCHARD- Advancing Global OCD Research Charity and ${ }^{6}$ Hertfordshire Partnership University NHS Foundation Trust, University of Hertfordshire, University of Cambridge

${ }^{\star}$ Corresponding author.

doi: 10.1192/bjo.2021.96

Aims. Obsessive Compulsive Disorder is a disabling and difficultto-treat condition, new treatment options are needed to improve health outcomes. Transcranial Direct Current Stimulation, a non-invasive form of neurostimulation, has shown positive results in a small number of studies as a safe and potentially efficacious treatment for OCD. There nevertheless remains uncertainty about the optimal stimulation protocol, magnitude and duration of effect, acceptability, tolerability and practicality of applying tDCS clinical settings. As existing data are inadequate to support a full-scale trial, we will deliver a feasibility study to address key research questions and knowledge gaps to enable the design and the development of the most efficient, cost effective, definitive trial.

Method. We designed Feasibility And Acceptability Of Transcranial Stimulation In Obsessive Compulsive Symptoms (FEATSOCS), a double-blind, sham-controlled, cross-over randomised multicentre study in 25 adults with OCD. We will stimulate the two most promising cortical sites, the orbitofrontal cortex (OFC) and the supplementary motor area (SMA). Each participant will receive three courses of tDCS (SMA, OFC and sham), randomly allocated, given in counterbalanced order. Each course comprises four 20 minutes-stimulations, delivered over two consecutive days, separated by at least four weeks' washout period. Blinded raters will regularly assess clinical outcomes before, during and up to four weeks after stimulation using validated scales. We will include relevant neurocognitive tasks, testing cognitive flexibility, motor disinhibition, cooperation and habit learning.

Result. FEATSOCS trial is currently underway and recruiting. Owing to the impact of COVID-19, a recruitment extension has been granted. At the study end, we will analyse the feasibility outcomes, magnitude of the effect of the interventions on OCD symptoms alongside the standard deviation of the outcome measure to estimate effect size, and determine the optimal stimulation target. We will also measure the duration of the effect of stimulation, to provide information on spacing treatments efficiently. We 
will evaluate the usefulness and limitations of specific neurocognitive tests to determine a definitive test battery. Qualitative data will be collected from participants to better understand their experience of taking part in a tDCS intervention, the impact on their overall quality of life and their views on the potential of tDCS as home based-intervention.

Conclusion. Further evidence is needed to establish whether tDCS could join the treatment armamentarium of OCD. The clinical outcomes in FEATSOCS will enable to further refine the methodology to ensure optimal efficiency in terms of both delivering and assessing the tDCS in OCD in a full scale trial.

The funder for this study is the National Institute for Health Research Programme, Research for Patient Benefit (RfPB) [Ref. no PB-PG-1216-20005]. Extra funding to allow study extension was provided by Orchard OCD. This study has received full ethics committee approval and protocol amendments approval form the Cambridge and Hertfordshire NHS Research Ethics Committee, IRAS Project ID 254507, REC ref: 19/EE/0046.

\section{Attitudes of medical students to electroconvulsive therapy \\ Patrick Clements* and Aidan Turkington \\ Belfast Health \& Social Care Trust \\ ${ }^{\star}$ Corresponding author.}

doi: 10.1192/bjo.2021.97

Aims. This study explores the different attitudes among fourth year medical students in Queen's University Belfast to Electroconvulsive Therapy (ECT) and investigates whether these are influenced by teaching and exposure to ECT during their undergraduate psychiatry placement. In particular we sought to determine firstly, correlates of baseline attitudes to ECT and secondly, whether specific forms of ECT teaching improved attitudes to ECT during their placement.

Method. This study was conducted in Queen's University Belfast and agreed with their ethics committee. Participants completed a questionnaire at the beginning of their psychiatry placement and another questionnaire in the second half of their placement. The first questionnaire captured background information and baseline attitudes. The second questionnaire recorded the educational and clinical experience gained on ECT during placement (for example lectures, tutorials, informal teaching, observing ECT and interacting with ECT patients), in addition to attitudes to ECT at this timepoint. Attitudes to ECT were assessed on a 5-point Likert scale. A positive attitude to ECT was defined as scoring agree/strongly agree on a 5-point Likert scale to the statement "I would recommend ECT for a patient if clinically indicated".

Result. 187 students were interviewed at both time points. At the outset of the psychiatry placement $66 \%$ of students reported a positive attitude to ECT. Positive attitude was associated with age: $72 \%$ of students under 24 had a positive attitude to ECT vs $58 \%$ of students over $24(\chi 2=3.5 ; \mathrm{P}<0.05)$. Of students who had previously attended a lecture on ECT $(n=117) 83 \%$ had a positive attitude to ECT vs $42 \%$ of those who had not previously attended a lecture $(\chi 2=33.5 ; \mathrm{P}<0.001)$.

Attitudes to ECT significantly improved during the placement ( $66 \%$ vs $94 \%$ positive; $\mathrm{t}=7.97 ; \mathrm{P}<0.001$ ). Students who attended a lecture on ECT during the psychiatry placement were more likely to have a positive shift in attitude ( $67 \%$ vs $49 \% ; \mathrm{F}=6.0$; $\mathrm{P}=0.01$ ). No other specific teaching modality was associated with a positive shift in attitude.
Conclusion. We conclude that undertaking a Psychiatry placement and particularly having a lecture on ECT significantly improves attitudes of medical students to ECT. It is therefore important that lectures on ECT are included in the medical undergraduate curriculum to allow students to be accurately informed about this essential treatment for a number of psychiatric disorders.

\section{Is attachment style in early childhood associated with mental health difficulties in late adolescence? \\ Philippa Clery ${ }^{\star}$, Angela Rowe, Marcus Munafò and Liam Mahedy University of Bristol \\ ${ }^{*}$ Corresponding author.}

doi: 10.1192/bjo.2021.98

Aims. Identifying factors that contribute to mental health difficulties in young people as early in life as possible are needed to inform prevention strategies. One area of interest is attachment. Although existing research has suggested an association between insecure attachment styles and mental health difficulties, these studies often have small sample sizes, use cross-sectional designs, and measure attachment as a discrete variable at a single point or use romantic relationship attachment as a proxy for childhood attachment. It is also unclear whether these associations persist into late adolescence. In this large prospective study we aimed to determine whether an insecure attachment style measured at repeated points in early childhood, is associated with depression and self-harm at 18 years.

Method. We used data from the Avon Longitudinal Study of Parents and Children cohort. Mothers completed attachment related questionnaires when their child was 18, 30, and 42 months old. Offspring depression and lifetime self-harm was assessed at 18 years in clinic using the Clinical Interview Schedule-Revised. Attachment was derived as a continuous latent variable in a structural equation modelling framework. Logistic regression was performed on participants with complete attachment data $(\mathrm{n}=7032)$ to examine the association between attachment style and depression and self-harm, with adjustment for potential confounders. Differential dropout was accounted for using multiple imputation.

Result. We found some evidence for an association between a more insecure attachment style in childhood, and a diagnosis of depression and life-time self-harm at age 18. In the fully adjusted imputed model, a one standard deviation increase in insecure attachment was associated with a $13 \%$ increase in the odds of depression $(\mathrm{OR}=1.13 ; 95 \% \mathrm{CI}=1.00$ to 1.27$)$ and a $14 \%$ increase in the odds of self-harm at age $18(\mathrm{OR}=1.14 ; 95 \% \mathrm{CI}=1.02$ to 1.25), for children who had more insecure attachment in early childhood, compared with children who had more secure attachment.

Conclusion. This is the largest longitudinal study to examine the prospective association between childhood attachment and depression and self-harm in late adolescence. Our findings strengthen the evidence suggesting that a childhood insecure attachment style is associated with mental health difficulties in late adolescence. Policies and interventions to support parenting behaviours that foster the development of secure attachment styles, or attachment-based therapies to improve attachment quality, could help reduce depression and self-harm in adolescence/ young adulthood.

Philippa Clery is supported by the Elizabeth Blackwell Institute for Health Research at the University of Bristol and the Wellcome Trust Institutional Strategic Support Fund. 